\title{
Deregulated ephrin-B2 expression in the mammary gland interferes with the development of both the glandular epithelium and vasculature and promotes metastasis formation
}

\author{
MIRJAM HALDIMANN $^{1 *}$, DOMENICA CUSTER $^{1 *}$, NADIA MUNARINI $^{1}$, \\ CHRISTOPH STIRNIMANN $^{1}$, GISELA ZÜRCHER ${ }^{1}$, VALERIA ROHRBACH ${ }^{1}$, \\ VALENTIN DJONOV $^{2}$, ANDREW ZIEMIECKI ${ }^{1}$ and ANNE-CATHERINE ANDRES ${ }^{1}$ \\ ${ }^{1}$ Department of Clinical Research, University of Bern, Tiefenaustrasse 120, CH-3004 Bern; \\ ${ }^{2}$ Anatomy Unit, University of Fribourg, Rue A.-Gockel 1, CH-1700 Fribourg, Switzerland
}

Received February 24, 2009; Accepted April 20, 2009

DOI: 10.3892/ijo_00000364

\begin{abstract}
Eph receptor tyrosine kinases and their membranebound ephrin ligands play key roles during morphogenesis and adult tissue homeostasis. Receptor-ligand interactions result in forward and reverse signalling from the receptor and ligand respectively. To delineate the role(s) of forward and reverse signalling in mammary gland biology we have established transgenic mice exhibiting mammary epithelial-specific overexpression of either the native ephrin-B2 or a dominant negative ephrin-B2 mutant incapable of reverse signalling. During pregnancy and lactation overexpression of the native ephrin-B2 resulted in precocious differentiation, whereas overexpression of mutated ephrin-B2 caused delayed epithelial differentiation and in disturbed tissue architecture. Both transgenes affected also mammary vascularisation. Whereas ephrin-B2 induced superfluous but organised capillaries, mutant ephrin-B2 overexpression resulted in an irregular vasculature with blind-ending capillaries. Mammary tumours were not observed in either transgenic line, however, the crossing with NeuT transgenic animals revealed that mutated ephrin-B2 expression significantly accelerated tumour growth and imposed a metastatic phenotype.
\end{abstract}

\section{Introduction}

Unlike other organs, the mammary gland develops mainly in the juvenile and adult organism. With the onset of ovarian

Correspondence to: Dr Anne-Catherine Andres, Department of Clinical Research, University of Bern, Tiefenaustrasse 120, CH-3004 Bern, Switzerland

E-mail: anne-catherine.andres@dkf.unibe.ch

*Contributed equally

Key words: angiogenesis, carcinogenesis, dominant negative mutant, Eph-ephrin forward-reverse signalling, mammary epithelium, metastasis function at puberty, the rudimentary epithelial anlagen are induced to proliferate and invade the surrounding fatty tissue giving rise to the primitive epithelial ductal tree characteristic of the virgin gland. During pregnancy the mammary epithelium expands drastically and differentiates until the entire gland is filled with secretory epithelium producing milk to nourish the young. After weaning, the mammary epithelium regresses by massive apoptotic cell death returning to the resting, cycling state in readiness for the next pregnancy (1). Thus, tissue composition and state of differentiation change drastically according to functional requirements, the end-differentiated state being reached only upon demand. These drastic upheavals also involve the mammary endothelium, which undergoes angiogenic expansion during pregnancy and lactation followed by ordered regression during involution (2). These developmental processes are regulated by complex cell-cell and cell-matrix interactions, as well as local paracrine and endocrine interactive stimuli. Thus, morphogenesis is a constant theme throughout the mammary life cycle making this organ an excellent model to study embryonic inductive principles maintained in the adult. Ironically, the complex life cycle of the mammary gland renders this organ especially prone to malignant transformation (3).

The functional unit of the mammary gland, the parenchyma, is composed of secretory and ductal epithelial cells, myoepithelial cells and pluripotent stem cells and is embedded in the mesodermal stroma. Proper functional and spatial development of the mammary gland is strictly dependent on mesenchymal-parenchymal interactions and the inductive role of the mesenchyme is reflected by the presence of paracrine factors in the stroma, modulating epithelial morphogenesis (4). It has long been established that the differentiative pathway of mammary epithelial cells depends on their position within the ductal system, however, the determinants of positioning and ductal pattern formation are largely unknown (5). Members of the wnt and Hox gene families, which specify developmental pathways during embryonic development, are also expressed in the adult mammary gland in a developmental stage-specific manner $(6,7)$. Furthermore, the Eph receptor tyrosine kinases and their 
ligands the ephrins have been implicated not only in a variety of morphogenic processes during embryonic development (8) but also in the maintenance of adult tissue homeostasis, including the development of the mammary gland (9).

The Eph receptors and ephrin ligands are membrane-bound proteins whose interaction requires direct cell cell contact. The ephrin ligands fall structurally into two classes: the ephrin-As are linked to the cell membrane by a GPI-tail and the ephrin-Bs are true transmembrane proteins. The Eph receptors, to date the largest family of receptor tyrosine kinases, are classified according to their prefered ligand interaction coorespondingly into EphA and EphB sub-families. Upon ligand interaction the receptors undergo autophosphorylation of the intracellular kinase domain initiating downstream signalling cascades, or forward signalling. Although the ephrins possess no obvious catalytic activity they also activate intracellular signalling pathways, a process referred to as reverse signalling. In the case of ephrin-B receptors, reverse signalling is initiated by phosphorylation of conserved tyrosine residues in the intracellular domain (10). Thus, interaction of receptor and ligand can provoke bi-directional signalling and mutual cell-cell communication. Termination of cell interaction and signalling can be achieved by different mechanisms. It has been shown that both, receptor and ligand are associated intracellularly with phosphatases capable of reverting phosphorylation (11). In addition, binding of EphA receptors with ephrin-A ligands can activate an extracellular metalloprotease, ADAM10, which cleaves the ligand and enables disengagement of the cells (12). Finally, it has been shown that termination of EphB and ephrin-B interaction can be achieved by bi-directional endocytosis of ligand-receptor complexes $(13,14)$.

Although a certain preference of receptor-ligand interaction has been observed, both receptor and ligands are promiscuous and can interact with several partners albeit with different affinities. Indeed, there is growing evidence that A class receptors can also interact with B-class ligands and vice versa (15). In addition, both receptors and ligands can activate and can be activated by a variety of other cell surface molecules. In particular, prominent cross-talk has been described between Eph/ephrin and E-cadherin or integrin molecules (16). Considering the large variety of molecular interactions, it is not surprising that the cellular responses observed upon receptor or ligand interaction show a broad diversity ranging from cellular adhesion to repulsion. A common theme, however, appears to be the modulation of adhesive and/or migratory properties of cells by targeting the cytoskeleton (15).

The observation that Eph receptors and their ligands exhibit reciprocal expression patterns during embryonic development has suggested that these molecules play an important role during embryonic development. Indeed, Eph and ephrin family members have been shown to be involved in a variety of morphogenic processes such as gastrulation, segmentation and establishment of tissue boundaries (17). In particular, EphB4 and ephrin-B2 have been shown to be instrumental in the formation of the embryonic vasculature (18). In contrast, less is known about the function(s) of Eph and ephrin family members in the adult. Some members are expressed in adult organs such as mammary gland, kidney, lung and at sites of neo-vascularization $(19,20)$, others control cell fate along the crypt-vilus axis in the small intestine (21) as well as bone (22) and blood homeostasis (23) and several members have been implicated in tumour development through their capacity of stimulating vascularisation (24).

The EphB4 receptor was originally isolated from the mature mouse mammary gland (19). Expression studies revealed that in the mammary parenchyma EphB4 expression was developmentally regulated and hormone-dependent, whereas the expression of its cognate ligand ephrin-B2 was less restricted (25). Overexpression of EphB4 in the mammary epithelium of transgenic mice interfered with the epithelial growth response and resulted in disturbed tissue architecture. Moreover, EphB4 expression imposed an aggressive and metastasizing phenotype on NeuT induced cancer cells (26). In order to investigate the role of ephrin-B2 in mammary gland development we have established two transgenic mouse lines overexpressing either the native ephrin-B2 gene or a dominant negative mutant of ephrin-B2 incapable of reverse signalling. These transgenic lines have allowed us to investigate the contribution of forward and reverse signalling in the maintenance of tissue homeostasis in the mammary gland. We demonstrate that expression of the two transgenes in the mammary epithelium exert distinct effects on tissue architecture, vascularization and carcinogenesis. These results suggest that correct ephrin-B2 reverse signalling is indispensable for the maintenance of tissue integrity in the mammary gland.

\section{Materials and methods}

Establishment of transgenic mice. An expression cassette consisting of the $1.2 \mathrm{~kb}$ MMTV-LTR promoter, a $0.2 \mathrm{~kb}$ fragment comprising the SV40 splice donor/acceptor sequence and a $0.3 \mathrm{~kb}$ fragment encoding the SV40 late polyadenylation site was used for construction of the transgenes. This expression cassette has previously been used successfully for the establishment of transgenic mice (26). A fragment of the mouse ephrin-B2 cDNA from position 1 to 1440 depleted of the polyadenylation signal was inserted between the splice donor acceptor sequence and the SV40 polyadenylation signal. To construct the mutant ephrin-B2 transgene, the ephrin-B2 cDNA cloned in pBluescript was digested with Pst I and religated. By means of this restriction digestion a fragment from position 1055 to 1428 was excised thereby eliminating the sequence encoding the intracellular conserved tyrosines but leaving the sequence encoding transmembrane region and part of the intracellular domain (position 856-918) intact. An oligonucleotide encoding three in frame stop codons was cloned into the Pst-site and the entire mutated cDNA was cloned as a Not I fragment into the expression vector. This cDNA encodes a C-terminally truncated ephrin-B2 protein missing the last 36 amino acids. The ephrin-B2 transgenic mice have been established commercially by pronuclear injection of fertilized eggs from a F2 C57B16xDBA/2 hybrid cross (Animal Facility of the Centre for Molecular Biology, University of Heidelberg, Germany). The mutated ephrin-B2 transgenic mice have been established in the same manner at the animal facility of the Swiss Institute for Experimental Cancer Research (ISREC), Lausanne. Mouse tail DNA was prepared as described (27) and transgenic mice were identified 
by PCR using oligonucleotides encompassing position 560-803 (5': ${ }^{\prime}$ AGGAATCACGGTCCAACAAG ${ }^{3} ;$;': ${ }^{\prime}$ GTCTCCTGC GGTACTTGAGC ${ }^{3^{\prime}}$ ) of the ephrin-B2 cDNA. Since in the endogenous gene the primer recognition sequences are separated by an $11450 \mathrm{bp}$ long intron, amplification of a $243 \mathrm{bp}$ long fragment was specific for the transgenes. From each construct two independent lines were obtained. Transgenic mice were crossed over 6 generations with inbred C57/B16 mice (Charles River, Wiga, Sulzfeld, Germany) in order to reduce complexity of the genetic background. These transgenic animals were bred to homozygosity. This was confirmed by Southern blotting and biologically by determining the pattern of inheritance. MMTV-LTR-NeuT transgenic mice of the FBV strain were obtained commercially (Charles River). Tumour development was followed in the F1 generation of FBV x C57/ B16 crosses.

RNA and protein analysis. For RNA and protein analysis, mammary glands were snap-frozen in liquid nitrogen and stored at $-70^{\circ} \mathrm{C}$ until required. RNA preparation, Northern blotting and RT-PCR were done, as described previously (26). The primers used to detect the native ephrin-B2 transgene were directed to position 1113 in the ephrin-B2 cDNA ( ${ }^{\prime}$ TACCACTAAGGACTGCAGAC ${ }^{3}$ ) and position 100 of the SV40 polyadenylation site $\left({ }^{5}\right.$ GTGGTTTGTCCAAACT $\mathrm{CATC}^{3}$ ), yielding a specific fragment of $430 \mathrm{bp}$. For detection of the truncated ephrin-B2 RNA, the 5' primer above was replaced by a primer directed against position 560 of the ephrin-B2 cDNA ( ${ }^{\prime}$ AGGAATCACGGTCCAACAAG ${ }^{3}$ ) yielding a specific fragment of $560 \mathrm{bp}$. Endogenous ephrinB2 expression was analyzed using the same primer pair as for the analysis of tail DNA. The PCR product was verified by cloning and sequencing. The absence of contaminating DNA was controlled by conventional PCR. For protein analyses, frozen tissue was macerated in SDS-PAGE sample buffer, boiled and subjected to Western blot analysis. Protein concentration was determined visually by amido black staining of $5 \mu 1$ protein extracts spotted onto nitrocellulose and equal loading was confirmed by coomassie blue staining of the Western blot filters. The production of the anti-mouse ephrin-B2 antibody has been described previously (25) and Western blotting was carried out by conventional methodology (28).

Histological analyses. Animals were routinely injected intraperitoneally with $200 \mu \mathrm{g}$ per g body weight of bromodeoxyuridine (BrdU, Sigma, Buochs, Switzerland) in PBS $3 \mathrm{~h}$ before sacrifice. The histological examination and whole mount staining employing the fourth inguinal mammary gland was done as described (26). For semi-thin sections, tissue fragments of $\sim 1 \mathrm{~mm}^{3}$ were fixed in Karnowsky's solution and processed for electron microscopy. Sections $(1 \mu)$ were stained with toluidine blue. Immunohistochemistry was performed as described (25). The antisera specific for mouse ephrin-B2 and CD31 were raised in our laboratory (25), whereas the other antisera were obtained commercially: anti-BrdU (Roche Diagnostics, Rotkreuz, Switzerland) anti-E-cadherin and anti- $\beta$-catenin (BD Transduction Laboratories, BecktonDickinson, Basel, Switzerland.), anti-smooth muscle actin (Sigma).
Mercox corrosion casting. Corrosion casts were prepared from the fourth inguinal mammary gland, as described previously (29). Briefly, the mammary gland was perfused with methylmethacrylate resin (Mercox, Vilene Hospital, Japan) containing $0.1 \mathrm{ml}$ accelerator per $5 \mathrm{ml}$ of resin. After hardening, the mammary glands were removed and the tissue components dissolved by $\mathrm{KOH}$ treatment. Samples were mounted, coated with gold and viewed using a Philips XL 30 FEG scanning electron microscope.

Determination of vascular densitiy. Blood vessels were visualized immunohistochemically on sections of lactating mammary glands using an anti-Von Willebrant factor-specific antibody (Roche Diagnostics), as described above. Blood vessels were counted on 5 consecutive visual fields adjacent to the skin using $\mathrm{x} 20$ magnification. For each experimental group 10 animals were evaluated.

\section{Results}

Characterization of the ephrin-B2 transgenic mouse lines. As an approach to investigate the role of ephrin-B2 in mammary gland development and function we have established two transgenic mouse lines overexpressing either the native ephrin-B2 cDNA or a 3' truncated dominant negative ephrin-B2 mutant (Fig. 1A). The resulting mutant protein lacks the $\mathrm{C}$ terminal 36 amino acids which include the 5 conserved tyrosine residues and the PDZ-binding motif. This reverse-signalling deficient mutant ephrin-B2, however, encodes the extracellular moiety and the transmembrane domain thereby allowing membrane localization of the protein and receptor engagement. Both the native and mutant ephrin-B2 cDNAs were placed under the control of the MMTV-LTR promoter which confers expression predominantly to the mammary gland of pregnant and lactating mice (30).

The two transgenic mouse lines were bred to homozygosity and analyzed for transgene expression during mammary gland development (Fig. 1B and C). RT-PCR analysis of RNA prepared from mammary glands of age matched control and transgenic animals revealed that transgene expression was induced during puberty, persisted until $~ 10$ weeks of age and declined thereafter. Transgene expression was again detected in late pregnancy and increased strongly thereafter. During these developmental stages the expression of the endogenous ephrin-B2 was, in comparison, modest. The strong transgene expression was maintained throughout lactation and declined rapidly after weaning (Fig. 1B). These expression patterns were also reflected at the protein level (Fig. 1C). The prominent expression of both transgene proteins, particularly of the mutant ephrin-B2, during late pregnancy and lactation suggested that phenotypic consequences could be expected. A direct Western blot comparison of the transgene proteins in extracts of lactating mammary glands confirmed the truncated nature of the mutant ephrin-B2 protein (Fig. 1D).

Transgene protein localisation in the mammary gland was investigated by immunohistochemistry. Whereas expression of the endogenous ephrin-B2 protein in lactating mammary glands of control females was below detection levels, both transgenes were strongly expressed in the lactating mammary epithelium and localized to the cell membrane (Fig. 1E). 
A

\begin{tabular}{|c|c|c|c|c|}
\hline & & & TM & PDZ \\
\hline MMTV-LTR & $\begin{array}{l}\text { SV } 40 \\
\text { Sp D D/A }\end{array}$ & ephrin-B2 & 1 & \begin{tabular}{l|l}
$Y_{5}$ & $S V 40 \mathrm{pA}$
\end{tabular} \\
\hline MMTV-LTR & $\begin{array}{l}S V 40 \\
S p D / A\end{array}$ & mutant ephrin-B2 & 11 & $S V 40 \mathrm{pA}$ \\
\hline
\end{tabular}

B


$\leftarrow N$

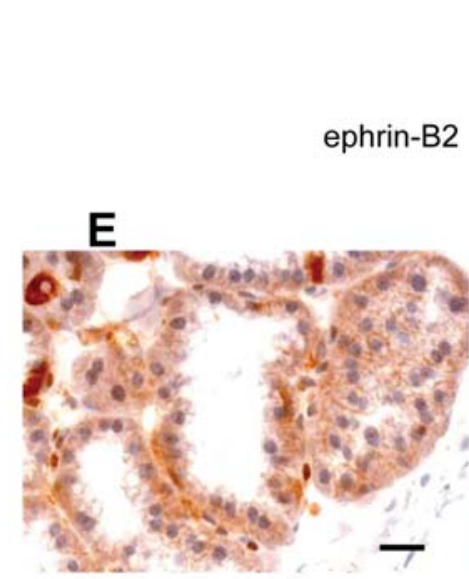

ephrin-B2

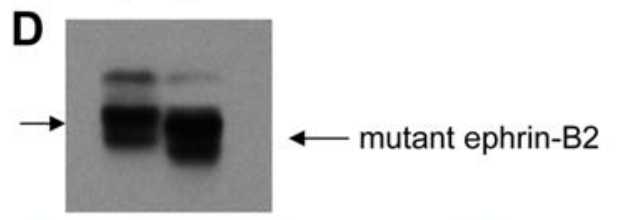

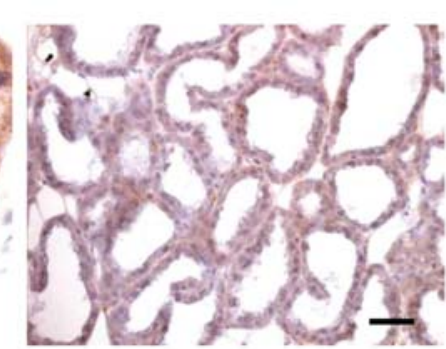

control

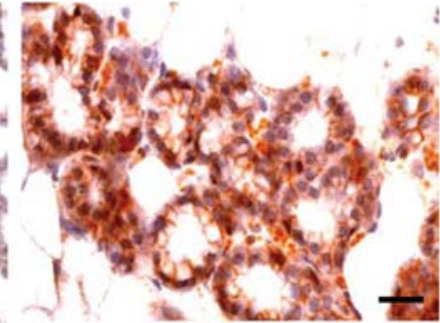

mutant ephrin-B2

Figure 1. Characterization of transgene expression. (A) Schematic representation of the ephrin-B2 and mutant ephrin-B2 transgene constructs. (B) RT-PCR analysis of endogenous ephrin-B2 expression (control), transgenic ephrin-B2 expression (ephrin-B2) and transgenic mutant ephrin-B2 expression (mutant ephrin-B2) in the respective control and transgenic lines throughout mammary gland development. (C) Western blot analysis of ephrin-B2 protein in control animals (control), ephrin-B2 transgenic animals (ephrin-B2) and mutant ephrin-B2 transgenic animals during mammary gland development. RNA or protein were prepared from mammary glands of 5-week $(5 \mathrm{w})$ and 10 -week (10w) old females, at day $13(13 \mathrm{~d} \mathrm{pr}), 16(16 \mathrm{~d}$ pr), and 18 (18 d pr) of pregnancy, at day $1(1 \mathrm{~d} \mathrm{lac})$ and $10(10 \mathrm{~d}$ lac) of lactation as well as at day $2(2 \mathrm{~d}$ inv) and 6 (6 d inv) of involution. (D) Size difference of the ephrin-B2 and mutant ephrin-B2 proteins analysed by Western blotting of protein extracts from lactating mammary glands of ephrin-B2 (1) and mutant ephrin-B2 (2) transgenic females. (E) Immunohistochemical localization of the transgenic ephrin-B2 (ephrin-B2) and mutant ephrin-B2 protein in lactating mammary glands. Sections were reacted with affinity purified ephrin-B2 antibodies and visualized with peroxidase coupled anti-rabbit IgG. Sections were counterstained with hematoxylin. Bars represent $25 \mu$ (ephrin-B2 and mutant ephrin-B2) and $50 \mu$ (control).

Transgene expression was patchy in appearance with highly expressing cells being located next to cells with low or no transgene expression.

Deregulated ephrin-B2 expression interferes with the development of the mammary epithelium. Since transgene expression was induced around puberty, we have used BrdU incorporation and whole mount staining of entire mammary glands to analyze transgene effects on the estrogen promoted outgrowth of the mammary epithelium. In control animals, the rudimentary epithelial anlagen began to proliferate in response to the onset of ovarian function at 3.5 weeks of age and strong proliferation could be detected at 4.5 weeks (Fig. 2A). At 10 weeks of age the entire fatty tissue was populated by branching ducts with numerous alveolar side structures and cell proliferation had ceased (Fig. 2D and G). In contrast, in both transgenic lines the epithelial outgrowth was severely delayed. Almost no cell proliferation was observed in the 

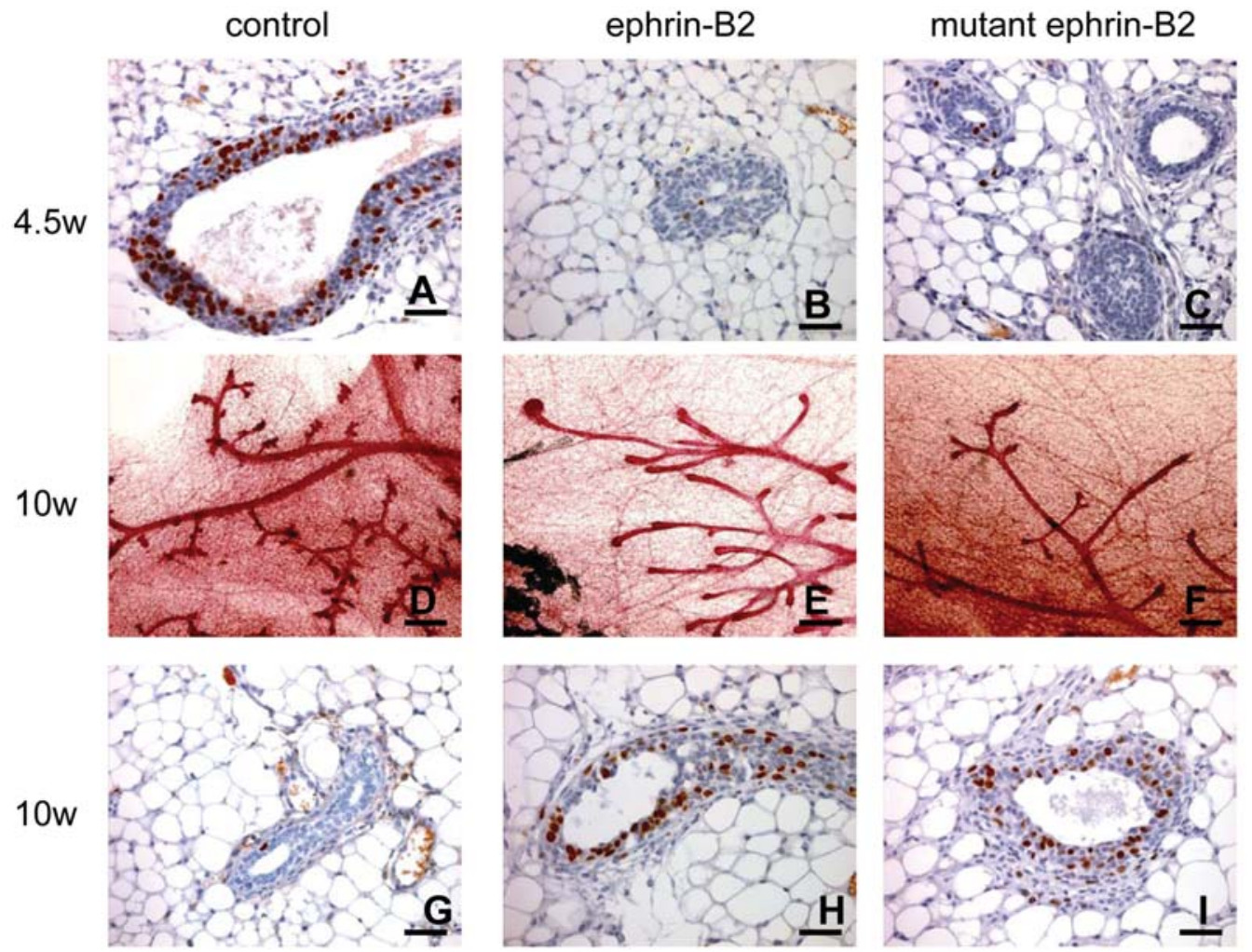

Figure 2. Branching morphogenesis of the mammary gland during puberty. Mammary glands of control (control), ephrin-B2 (ephrin-B2) and mutant ephrin-B2 (mutant ephrin-B2) transgenic females were analyzed by immunohistochemical detection of BrdU incorporation at the age of 4.5 weeks (4.5 w) (A-C) and 10 weeks (10 w) (G-I) and by whole mount staining at 10 weeks (D-F). Bars represent $100 \mu$ (D-F) and $25 \mu$ (A-C and G and H).

epithelial cells of 4.5-week old pubertal transgenic females (Fig. 2B and C). In agreement with this low proliferative activity, the mammary ducts had not reached the borders of the fatty tissue at 10 weeks of age. Moreover, the epithelial ducts exhibited an immature appearance, branching frequency was low and there were still many terminal end buds visible at the expense of alveolar side structures (Fig. 2E and F). Proliferation, however, commenced in older animals and could be readily detected in the epithelium of 10-week old transgenic females (Fig. 2H and I). Although the epithelial development in transgenic animals caught up to a certain extent with controls, transgenic virgin animals never exhibited a fully developed mammary gland (data not shown).

The major development of the mammary gland occurs during pregnancy when the mammary epithelium prepares for lactation by proliferation and differentiation. During this developmental stage transgene expression was strongly induced and distinct phenotypic consequences could be observed in the two transgenic lines. At day 16 of pregnancy the mammary epithelium of control animals revealed a high proliferative activity (Fig. 3A). The small lumen of the alveoli and the localization of the nuclei in the centre of the cells illustrate the low differentiation grade of the epithelium. In contrast, the mammary epithelium of ephrin-B2 transgenic females exhibited clear signs of differentiation with modest cell proliferation at day 16 of pregnancy (Fig. 3B). The alveoli exhibited a well developed lumen and the irregular appearance of the apical side as well as the material deposits in the lumen indicated that secretory activity had been initiated. By day 18 of pregnancy, proliferation had essentially ceased, differentiation had advanced and the alveolar morphology was comparable to that of the normal lactating state (Fig. 3E). In the mutant ephrin-B2 transgenic line no gross differences to control animals could be observed at day 16 of pregnancy (Fig. 3C), consistent with the observation that transgene expression was induced after this time point. In these females, however, the differentiation process at the end of pregnancy was severely compromised. At day 18 of pregnancy the indications of differentiation were completely absent in the epithelium of the mutant ephrin-B2 females and the morphology resembled the control mid-pregnant stage (Fig. 3F). Although the differentiation process in these animals did not seem to be initiated, cell proliferation was reduced compared to the controls (Fig. 3C and F).

Whole mount staining of mammary glands at day 18 of pregnancy served to illustrate the abnormal development of the mammary epithelial tree in the two transgenic lines (Fig. 3G-I). Compared to the mammary glands of control animals (Fig. 3G), the lobules of the mammary glands of females overexpressing ephrin-B2 consisted of more and larger alveoli. Furthermore, the lobules were located closer to the main ducts, indicating that the side branches did not invade deeply into the fatty tissue (Fig. 3H). In contrast, the mammary glands of the mutated ephrin-B2 transgenic females contained fewer lobules with smaller and compacter alveoli as compared to controls (Fig. 3I).

Deregulated ephrin-B2 expression interferes with tissue architecture and function in the lactating mammary gland. At lactation, the end-differentiated state of the mammary gland, 


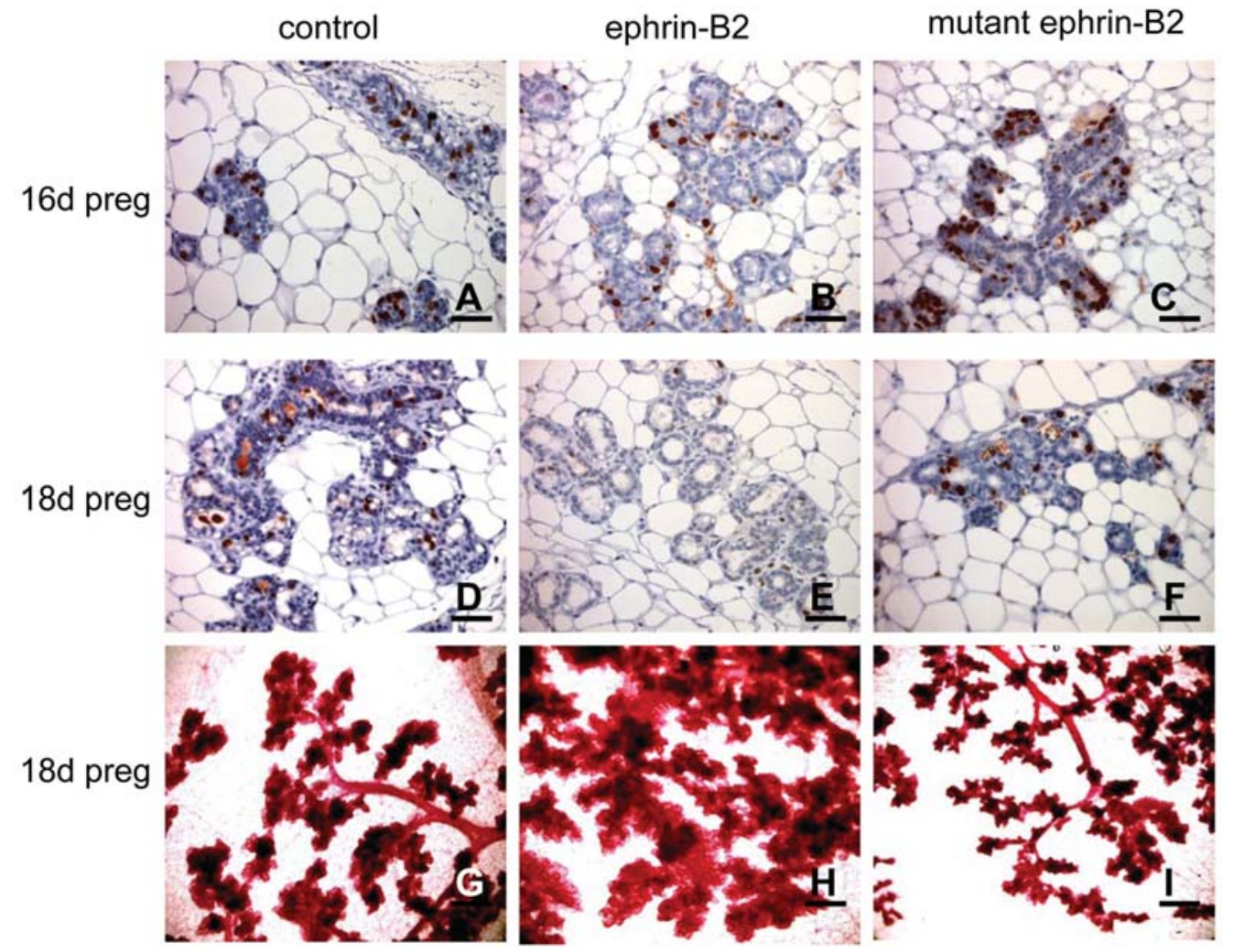

Figure 3. Mammary gland development during pregnancy. Mammary glands of control (control), ephrin-B2 (ephrin-B2) and mutant ephrin-B2 (mutant ephrin-B2) transgenic females were analyzed by immunohistochemical detection of BrdU incorporation (A-F) and whole-mount staining (G-I) at day 16 (16 d preg) and 18 (18 d preg) of pregnancy. Bars represent $50 \mu$ (A-F) and $100 \mu$ (G-I).

the entire fat pad is normally occupied by secretory epithelial alveoli (Fig. 4A). Since high transgene protein expression was observed in both transgenic lines during lactation, phenotypic consequences could be expected to be most evident during this developmental stage. Analysis of tissue morphology revealed that the lactating mammary glands of ephrin-B2 transgenic mice exhibited a well-organized structure. Although more fatty tissue remained in these glands than in those of control animals the alveolar units had a normal, functional appearance. Inspection of toluene blue stained semi-thin sections revealed very extensive secretory activity in mammary glands of ephrin-B2 transgenic animals. Specifically, the abundance of milk fat droplets in the alveolar lumen was significantly higher than in control animals (Fig. 4B). In contrast, lactating mammary glands of mutant ephrin-B2 mice exhibited an irregular morphology. The alveolar structures varied in size and exhibited a disturbed morphology. Regionally, the epithelial cells seemed to have lost contact with their neighbours and protruded into the lumen where apoptotic cells could be observed (Fig. 4C and O). Although some secretory activity was maintained, the low frequency of milk fat droplets indicates that secretion was impaired (Fig. 4C). This was also supported by the observation that mutant ephrin-B2 females were only able to nourish small litters (average 4-6 pups) and the pups often suffered from growth retardation. Northern blot analysis of milk protein gene expression revealed no significant differences in casein and whey acidic protein mRNA levels (data not shown) suggesting that the lactational handicap of the mutant ephrin-B2 females was not due to reduced expression of milk protein genes but rather to reduced secretory activity and fat droplet formation.

The secretory capacity of the mammary gland is dependent on the correct differentiation and polarization of the epithelial cells as well as on the presence of myoepithelial cells surrounding the alveoli. The presence and distribution of myoepithelial cells was assessed for by immunohistochemical staining of smooth-muscle actin. In the lactating mammary glands of control animals the myoepithelial cells appeared as a discontinuous layer surrounding and protruding into the epithelium (Fig. 4D). In the lactating mammary glands of ephrin-B2 transgenic animals the myoepithelial cells were normally arranged around the alveoli comparable to the control (Fig. 4E). In the lactating mammary glands of mutant ephrinB2 mice, however, the myoepithelium appeared highly irregular, the borders between epithelium and myoepithelium were not clearly delineated and the myoepithelial cells often appeared cuboidal rather than flattened (Fig. 4F).

E-cadherin is one of the major proteins involved in epithelial cell-cell contact and the establishment of a polarized phenotype. Intracellularly, $\beta$-catenin is a component of the protein complex connecting cadherin to the cytoskeleton. We have analyzed the presence and localization of these two proteins as an additional parameter of functional epithelial organization. In the lactating mammary glands of control animals, both proteins were localized to the lateral cell membranes (Fig. 4G and J). Similarly, E-cadherin and Bcatenin were mostly normally localized in the mammary glands of ephrin-B2 overexpressing animals. Only regionally 


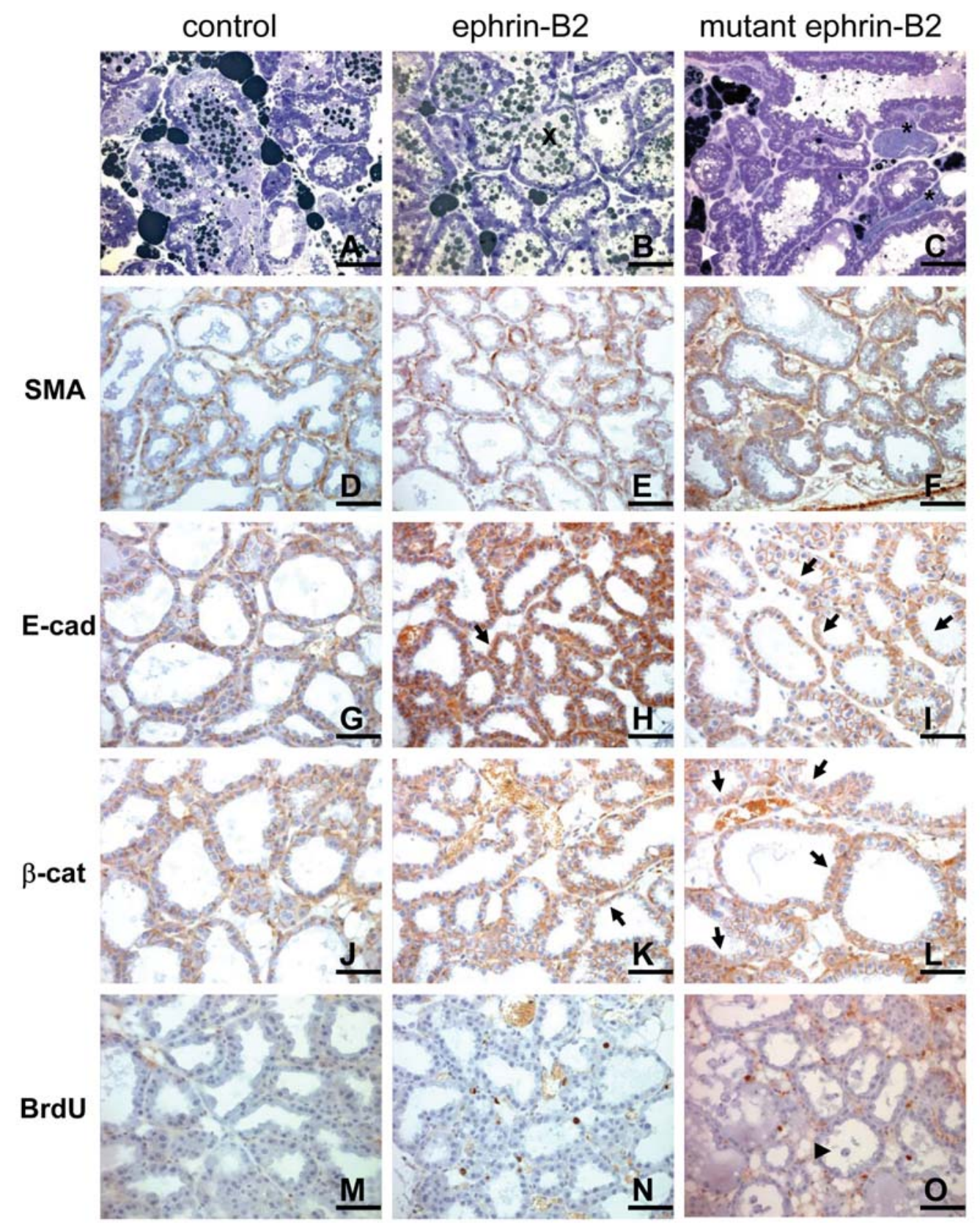

Figure 4. Tissue architecture of lactating mammary glands. (A-C) Sections $(1 \mu)$ of mammary glands of control (control), ephrin-B2 (ephrin-B2) and mutant ephrin-B2 (mutant ephrin-B2) transgenic females at day 10 of lactation were stained with toluene blue. (D-F) Myoepithelial cells were visualized by immunohistochemical staining of smooth muscle actin (SMA) in 4- $\mu$ sections of 10 days lactating mammary glands. (G-L) Mammary glands at day 10 of lactation were analyzed immunohistochemically for the presence of E-cadherin (E-cad) and B-catenin (ß-cat). (M-O) Cell proliferation was determined by immunohistochemical detection of BrdU incorporation (BrdU). Bars represent $50 \mu$. x points to fat droplets (B), "to blood vessels (C), (black arrow pointing to the right) to apoptotoc cells $(\mathrm{C}$ and $\mathrm{O}), \bullet$ to disconnected alveoli with protruding cells $(\mathrm{C})$ and arrows indicate abnormal protein localisation.

E-cadherin and B-catenin were homogeneously distributed in the cytoplasm (Fig. $4 \mathrm{H}$ and $\mathrm{K}$ ). Immunohistochemical detection of E-cadherin in lactating mammary glands of mutant ephrin-B2 transgenic mice irregular E-caherin and B-catenin localization was more frequent that in ephrin-B2 transgenic mice. In single alveoli, epithelial cells exhibiting correct lateral localization of E-cadherin were located next to cells with homogeneous cytoplasmatic localization (Fig. 4I). Similarly, B-catenin was often localized in the cytoplasm and occasionally even in the nucleus (Fig. 4L). These experiments indicate that predominantly mutant ephrin-B2 expression interferes with the parenchymal organisation and affects the homophilic interaction of the mammary epithelial cells.
In the lactating, differentiated state cell proliferation was rarely observed in the mammary glands of control animals (Fig. 4M). Interestingly, cell proliferation was frequently seen in lactating mammary glands of both transgenic lines (Fig. 4N and $\mathrm{O}$ ). BrdU incorporation was evident in alveolar epithelial cells as well as in extra-alveolar cells of myoepithelial or endothelial origin. These results indicate that during lactation the mammary glands of both transgenic lines exhibit an increased cellular turnover.

Deregulated ephrin-B2 expression interferes with vascular development and leads to tumour progression. EphB4 and ephrin-B2 have been shown to be intimately involved in the 
Table I. Formation of mammary tumours in transgenic females.

\begin{tabular}{lccc}
\hline Strain & Frequency & Latency & Metastasis \\
\hline NeuT & $15 / 15$ & $6.5 \pm 0.58$ months $^{\mathrm{a}}$ & $0 / 15$ \\
NeuT/ephrin-B2 & $12 / 12$ & $6.1 \pm 0.63$ months $^{\mathrm{a}}$ & $2 / 12$ lung \\
NeuT/mutant ephrin-B2 & $12 / 16$ & $1.5 \pm 0.78$ months $^{\mathrm{a}}$ & $10 / 12$ lung liver \\
NeuT/EphB4 & $22 / 24$ & $3.2 \pm 0.62$ months $^{\mathrm{a}}$ & $19 / 22$ lung \\
\hline
\end{tabular}

${ }^{\mathrm{a}}$ After first pregnancy.

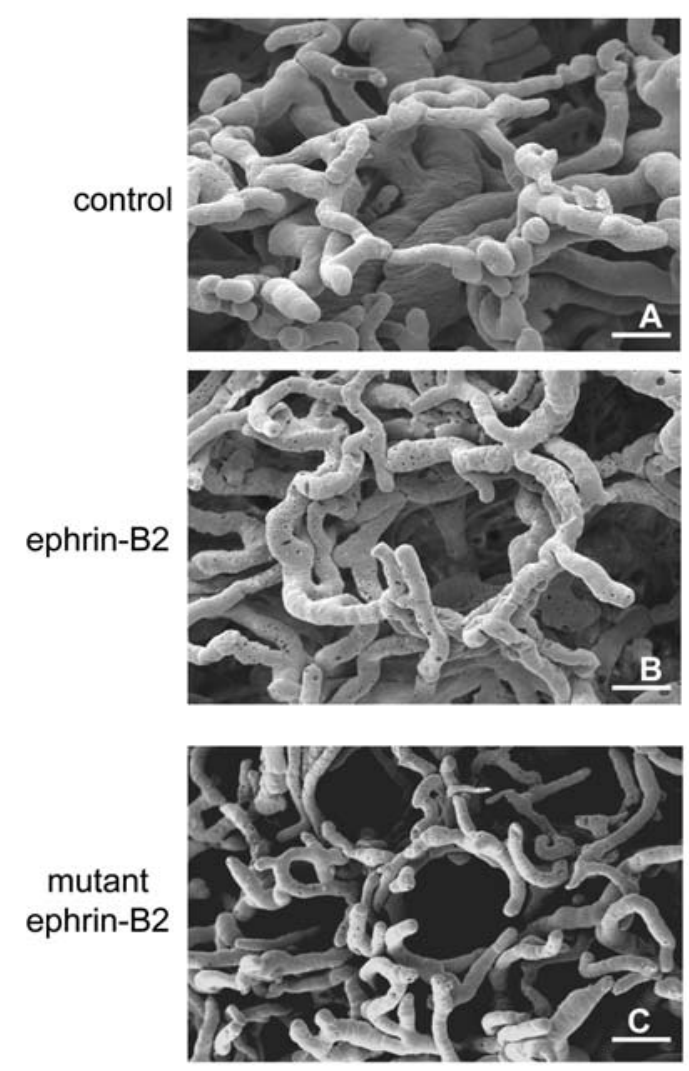

Figure 5. Mercox corrosion casts of the vasculature in mammary glands at day 10 of lactation from control (control), ephrin-B2 (ephrin-B2) and mutant ephrin-B2 (mutant ephrin-B2) transgenic animals. Bars represent $25 \mu$.

regulation of the vasculature predominantly during embryonic development but also at sites of neo-vascularisation in the adult. Histological examination of lactating mammary glands indicated a preponderance of erythrocytes in the interalveolar space predominantly in the mutant ephrin-B2 transgenic line (Fig. 4C). Moreover, proliferating endothelial cells could occasionally be observed in these glands (data not shown). This prompted us to investigate the extent and organization of the vasculature using corrosion casting followed by scanning electron microscopy. The vasculature in lactating mammary glands of control animals is composed of a well developed capillary network, the secretory alveoli being enveloped by single capillary honeycombs (Fig. 5A) (2). A similarly ordered capillary structure was observed in the ephrin-B2 overexpressing lactating mammary glands. However, in contrast to the control animals, the secretory alveoli were consistently enveloped by a bi- or even multilayered capillary plexus (Fig. 5B). This suggests that overexpression of ephrin-B2 promotes vascular development without interfering with vascular tissue architecture. Inspection of corrosion casts from mutant ephrin-B2 transgenic mammary glands revealed a completely different phenotype. In these glands a surplus of irregularly arranged capillaries was evident with numerous capillary stumps and blind-ending vessels (Fig. 5C). In addition, determination of the blood vascular density in lactating females revealed that in contrast to control animals and ephrin-B2 transgenic females, exhibiting a vascular density of $7.2 \pm 0.83$ and $9.4 \pm 1.14$, respectively, the number of blood vessels with $17.4 \pm 1.81$ was significantly increased in the mammary glands of mutant ephrin-B2 females. These data suggest that the inhibition of ephrin-B2 signalling disturbs the proper organization and extent of the capillary network.

Reduced tissue integrity and promotion of angiogenesis are hallmarks of tumour progression. We were therefore interested in the tumourigenic potential of the two transgenes. Mammary tumours have not been observed in either line even after multiple pregnancies and an observation period of 2 years. To assess a possible influence of deregulated ephrin-B2 expression on tumour progression, we have crossed these animals with mice bearing the NeuT oncogene under the MMTV-LTR promoter. In the C57/B16 genetic background all NeuT transgenic females developed non-invasive mammary adenocarcinoma with a latency interval of 6.5 months after their first pregnancy. In double transgenic females bearing the NeuT oncogene and the native ephrin-B2 cDNA, both the frequency and latency time remained unchanged. In 2 out of 12 animals metastases in the lung were observed (Table I). Interestingly, in double transgenic mice expressing the NeuT and the mutated ephrin-B2 gene, tumour development was significantly altered. In these animals tumour penetrance was only $75 \%$, however, if tumours developed they appeared after a much shorter latency of 1.5 months. Moreover, in almost all animals the tumours rapidly metastasized to the lungs. In two of these females additional metastases in the liver were observed (Table I). We have previously described a similar effect of EphB4 overexpression on tumour progression. As in the NeuT/mutant ephrin-B2 mice, NeutT/EphB4 double transgenic females developed metastasizing tumours to the lung after a reduced latency (26). In these animals, however, liver metastases have never been observed. The histological appearance of the primary tumours and the metastases of 



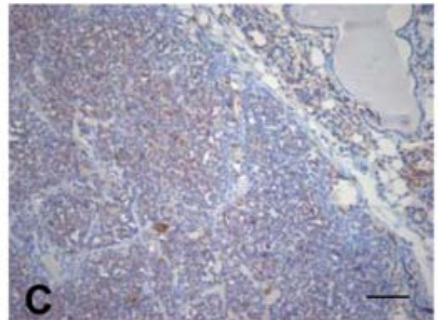

Primary tumor CD31

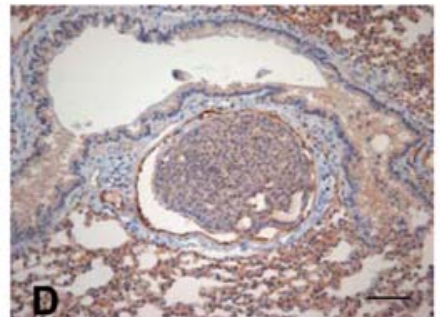

Lung metastasis CD31

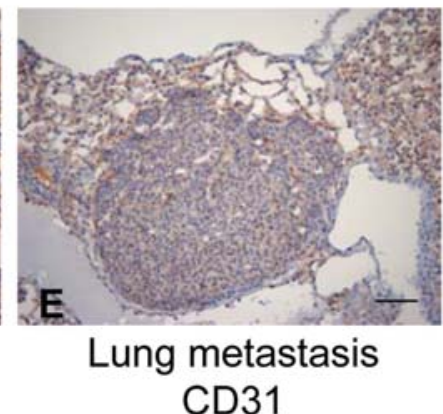

Figure 6. Histological appearance of primary mammary tumors and lung metastases in mutant ephrin-B2 trangenic mice. Hemtatoxylin/eosin staining of the primary tumor (A) and its metastasis (B). Immunohistochemical detection of CD31 in the primary tumor (C) and two of its metastatic nodules in the lung (D and E). Bars represent $50 \mu$.

mutant ephrin-B2 mice was similar, although metastases often exhibited necrotic cells in the center of the nodule (Fig. 6A and B). Interestingly, immunohistochemical staining with the blood endothelial marker anti-CD31 revealed that the metastases grew within blood vessels (Fig. 6D) as well as within the lung alveolar tissue (Fig. 6E). Intravascular tumour growth was never observed in the primary mammary tumours (Fig. 6C). These results indicate that overexpression of EphB4 or inhibition of ephrin-B2 signalling can impose an invasive phenotype on transformed but non-invasive tumour cells.

\section{Discussion}

As an approach to elucidate the function of ephrin-B2 in the biology of the mammary gland and to asses the role of forward and reverse signalling we have generated two complementary transgenic mouse lines. The first line exhibits overexpression of the native ephrin-B2 in the mammary epithelium predominantly during pregnancy and lactation. The transgene protein is identical to the endogenous protein and is capable of inducing forward and reverse signalling, of interacting with other signalling partners and of being properly processed upon receptor interaction. In contrast, the second line carries a dominant negative ephrin-B2 mutant which lacks the Cterminal 36 amino acids. This truncation removes not only the conserved tyrosines known to be crucial for reverse signalling, but also the PDZ domain involved in the formation of phosphorylation-dependent and -independent signalling complexes (16). The mutant protein retains, however, the transmembrane domain and the major portion of the intracellular domain. Similar dominant negative ephrin-B mutants have been successfully used to dissect the cellular responses to forward and reverse signalling in vivo and in vitro. These ephrin-B2 mutant constructs, however, were more extensively truncated (31-33). Thus, we cannot exclude that our minimally truncated mutant is still capable of restricted signalling involving so far uncharacterised intracellular amino acid motifs. It has been shown that termination of receptor ligand interaction can be achieved by internalization of ligand-receptor complexes. The correct bi-directional internalization was shown to be dependent on the presence of the intracellular domain of the ephrin molecule, however, the sequence responsible was not identified $(13,14)$. We cannot formally exclude that our minimally truncated mutant can still be properly internalized and processed. In summary, our ephrin-B2 mutant is expected to induce forward signalling but to be unable to elicit at least the phosphorylation- and PDZ-dependent reverse signalling. Thus the two transgenic lines allowed us to compare the phenotypic consequences of over-stimulated forward signalling with or without concomitant reverse signalling.

\section{Ephrin-B2 is involved in the development of the mammary} epithelium. The mammary glands of the transgenic females were characterized by an altered development of the mammary epithelium. During the estrogen-induced growth phase at puberty, a severe retardation of the mammary epithelial outgrowth and low branching activity was observed in both lines. Since both lines, the reverse signalling competent and incompetent, were similarly affected, we conclude that this effect is brought about by an over stimulation of forward signalling. This hypothesis is supported by the fact that a similar phenotype was observed in females overexpressing EphB4 in the mammary epithelium (26). Estrogen is a hormonal master regulator of mammary epithelial outgrowth and development. This hormone, however, acts indirectly on 
the epithelial target cells by mechanisms which in their complexity are not fully understood (34). We have previously shown that estrogen induces EphB4 expression in the mammary gland whereas the expression of ephrin-B2 is less affected by this hormone (25). In contrast to most receptor tyrosine kinases, Eph receptors generally lack mitogenic activity and suppress proliferation (35). It is conceivable that EphB4 serves as a negative regulator of mammary epithelial outgrowth and attenuates the estrogen induced growth phase.

The second massive development of the mammary epithelium occurs during pregnancy, where progesteroneinduced side branching and alveolar differentiation prevails. In this growth phase, distinct phenotypic consequences of transgene expression became evident. Whereas overexpression of native ephrin-B2 led to precocious differentiation of the epithelium, expression of mutated ephrin-B2 resulted in a reduced and delayed development of the epithelium. Opposite effects were also observed during lactation. Ephrin-B2 transgenic females exhibited well-organized mammary glands with prominent secretory activity. In contrast, the lactating mammary glands of females expressing the mutated ephrin-B2 were charcterized by disturbed tissue architecture and reduced secretional activity. This phenotype is very reminiscent of the observations made in transgenic mice overexpressing EphB4 in the mammary epithelium (26). This suggests that bi-directional or reverse signalling is required for the functional morphogenesis of the mammary epithelium and that uncontrolled forward signalling affects tissue organization and function. We cannot, however, exclude that the observed effects are not (only) brought about by direct EphB4-ephrinB2 interaction. The fact that endogenous EphB4 expression is low during lactation supports the notion that other mechanisms are also involved in the establishment of the observed phenotypes. Indeed, EphB4-independent mechanisms of ephrin-B2 action have been described, be it through interaction with other EphB or EphA receptors (15), cross-talk with other transmembrane receptors such as integrins or FGF receptors (16) or by constitutive interaction with PDZ-domain proteins (35). Although expression of Ephs and ephrins has not been comprehensively studied during mammary gland development, expression of EphB2 and EphA2 has been observed in mammary epithelial cells (19 and unpublished observation). Moreover, integrins and FGF receptors are acknowledged to play important roles in the development and functioning of the mammary gland $(36,37)$.

The homophillic cell adhesion molecules, the cadherins are another class of molecules important for mammary gland organization. E-cadherin is instrumental in the establishment of the polarized epithelial phenotype and is essential for the establishment of the differentiated functional state of the mammary epithelium during lactation (38). Moreover, differential expression of $\mathrm{E}$ - and $\mathrm{P}$-cadherin is responsible for the compartmentalisation of myoepithelial and epithelial cells (39). Interestingly, a subtle cross-talk between E-cadherin and the Ephs and ephrins has been described $(40,41)$. Disruption of the E-cadherin gene led to the down-regulation of EphA2 expression and to the induction of the expression of EphB2, EphB4 as well as of ephrin-B1 and ephrin-B2. This observation led to the suggestion that EphB-ephrin-B signalling is involved in out-growth and migratory processes rather than in cell anchoring (9). Thus it is tempting to speculate that deregulated expression of ephrin-B2 may influence the expression of E-cadherin. Indeed, in the lactating mammary glands of predominantly the mutant ephrin-B2 transgenic line disturbed expression and localization of E-cadherin and its intracellular or nuclear anchor ß-catenin was observed. Histologically these glands were characterized by a fragile morphology and abundant apoptotic cells in the alveolar lumen, a characteristic very reminiscent of the phenotype described in transgenic mice with a mammary epithelialspecific knock-out of the E-cadherin gene (42). Taken together these data suggest that inhibition of ephrin-B2 reverse signalling affects cell adhesion by interfering with proper E-cadherin function.

Deregulated epithelial ephrin-B2 expression interferes with vascular development. In addition to the epithelial structures, deregulated expression of ephrin-B2 in the lactating glands also affected the development of the mammary vasculature. Overexpression of native ephrin-B2 stimulated vascular development without interfering with its organisation, resulting in multi-layered capillary rings surrounding the epithelial alveoli. In contrast, the vasculature of lactating mammary glands of transgenic mice expressing the mutated ephrin-B2 was characterized by supernumerary capillaries many of which were apparently blind-ended. This suggests that the epithelial cell induced forward signalling in the endothelium is capable of stimulating the formation of blood capillaries, however, bi-directional signalling between these two cell populations is required for proper capillary organisation. It has been shown that non-endothelial expression of EphB receptors and ephrin-B ligands is essential for the modulation of vascular growth and capillary guidance during embryogenesis (43). Moreover, fibrin implants presenting the receptor binding domain of ephrin-B2 have been shown to stimulate the formation of capillaries in a chicken CAM model (44). In this study, however, the question of whether these capillaries form a functional network was not addressed. Given the importance of adequate blood supply for proper organ function we cannot exclude that part of the observed phenotypes in the mammary epithelium are a consequence of the disturbed vasculature.

Several knock-out mouse models created to dissect the role(s) of forward and reverse signalling in vascular formation have yielded contradictory results. Whereas one study documents the necessity of ephrin-B2 reverse signalling (33), another publication suggests that ephrin-B2 reverse signalling is not required for vascular development (31). These contradictory results may be explained by differences in the constructs used or the different experimental concepts (rescue of phenotype vs. induction of phenotype). Since in these experiments the ephrin-B2 mutation was effective in the entire organism, no conclusion can be made as to whether the observed phenotype was due to defective signalling in the endothelial cells and/or in the surrounding tissue. The different vascular phenotypes observed in our transgenic animals indicate the importance of ephrin-B2 reverse signalling in non-endothelial cells for the correct organization of the vasculature. However, we cannot conclude if the observed phenotypes are due to a direct interaction between the epithelial and endothelial cells or are mediated by an additional cell type 
such as myoepithelial or stromal cells. Although the endothelial and epithelial compartments are mostly separated by at least the basement membrane hindering direct cell-cell contacts, interactions between endothelial and epithelial cells have been shown to be crucial not only for vascular development but also for branching ductal-alveolar morphogenesis (45). In our transgenic animals endothelial-epithelial cell contacts may be facilitated by the disturbed arrangement of the myoepithelial cells thereby promoting the effects on the mammary endothelium. Alternatively, transgene expression might indirectly influence angiogenesis by inducing the expression of angiogenic factors in the epithelium itself or in the myoepithelium $(45,46)$.

Inhibition of ephrin-B2 reverse signalling promotes tumour progression. Ephs and ephrins are modulators of cell movement and pattern formation and are involved in the maintenance of tissue homeostasis. Thus, an important issue to address is the question whether they are also involved in carcinogenesis. We have not observed development of mammary tumours in the two ephrin-B2 transgenic lines, indicating that perturbed EphB4/ephrin-B2 signalling per se is not sufficient to induce cellular transformation. The crossing of these mice with MMTV-NeuT transgenic animals, however, revealed that the native and the mutant ephrin-B2 exerted distinct effects on the growth characteristics of NeuT induced mammary tumours. Whereas expression of the native ephrinB2 did not significantly alter the tumour latency and only minimally the tumour invasiveness, expression of the mutant ephrin-B2 led to rapid tumour development and a high metastatic potential. The observation that more metastatic nodules were found within vessels than within the alveolar tissue suggests that metastatic cells attach to and grow to the endothelium before seeding into the lung tissue. The consequences of mutant ephrin-B2 expression resembled closely the effects of EphB4 expression with respect to malignant growth (26). This indicates that unscheduled stimulation of forward signalling favours tumour progression. It seems that in the context of the mammary gland, ephrin-B2 expression in the epithelium exerts a surveillance function controlling the responses to EphB4 activation. This notion is further supported by our previous observation that the endogenous ephrin-B2 gene is down-regulated in c-myc or Ha-ras induced mouse mammary tumour cells (25).

It has been shown that expression of EphB4 on tumour cells promotes tumour growth by stimulation of vascularisation (47). The authors elegantly show that this effect is independent of the EphB4 kinase activity indicating that the reverse stimulation of endothelial cells is mainly responsible for this effect. Interestingly, results obtained in a mouse xenograft model indicate that the interaction of EphB4 on tumour cells with ephrin-B2 on adjacent tumour cells suppresses carcinogenic growth. In contrast, tumoural EphB4 expression in the absence of ephrin-B2 promotes tumour growth (48). Moreover, EphB4 expression promotes progression of ovarian cancer (49) and correlates in endometrial cancer with unfavourable prognosis and outcome of the disease $(50,51)$. In our transgenic mouse model induction of tumour progression was observed in mice overexpressing the reverse signalling defective ephrin-B2 mutant or the EphB4 receptor, whereas overexpression of the native ephrin-B2 gene had little effect. This suggests that tumour progression is favoured by overstimulation of EphB4 signalling only if the adequate ephrin-B2 reverse response is suppressed.

In addition to EpB4, EphA2, one of the few members of the Eph family expressed in adult epithelium, has been found to be up-regulated in various human and mouse cancers and expression of EphA2 has been detected on both tumour cells and endothelial cells of the tumour vasculature (52). Transfection of EphA2 into normal mammary epithelial cells was sufficient for transformation and this effect was independent of the receptor's kinase activity $(53,54)$. In contrast, the kinase activity of EphA2 receptors expressed in endothelial cells was required to promote tumour growth (55). These observations indicate that the mode of action of Eph and ephrin molecules drastically depends on the cell type they are expressed in, the cell type they are interacting with and the physiological setting in which this interaction takes place.

\section{Acknowledgements}

The authors wish to thank Robert Strange for helpful discussions and critical comments on the manuscript. This study was supported by the Swiss National Science Foundation (3100A0-109872), the Swiss Cancer League (OCS-0151002-2004 and KLS-02105-08-2007), the Schweizerische Stiftung für Klinisch-Experimentelle Tumorforschung and the Bernese Cancer League.

\section{References}

1. Richert MM, Schwertfeger KL, Rydner JW and Anderson SM: An atlas of mouse mammary gland development. J Mamm Gland Biol Neopl 5: 227-242, 2000.

2. Djonov V, Andres A-C and Ziemiecki A: Vascular remodelling during the normal and malignant life cycle of the mammary gland. Micr Res Tech 52: 182-190, 2001.

3. Russo J, Fu Hu Y, Silva IDCG and Russo IH: Cancer risk related to mammary gland structure and development. Micr Res Tech 52: 204-224, 2001.

4. Schmeichel KL, Weaver VM and Bissell M: Structural cues from tissue microenvironment are essential determinants of the human mammary epithelial phenotype. J Mamm Gland Biol Neopl 3: 201-214, 1998

5. Stingl J and Caldas C: Molecular heterogeneity of breast carcinomas and the cancer stem cell hypothesis. Nat Rev Cancer 7: 791-799, 2007.

6. Friedmann Y, Daniel CA, Strickland P and Daniel CW: Hox genes in normal and neoplastic mouse mammary gland. Cancer Res 54: 5981-5985, 1994.

7. Gavin BJ and McMahon AP: Differential regulation of the wnt gene family during pregnancy and lactation suggests a role in post-natal development of the mammary gland. Mol Cell Biol 12: 2418-2423, 1992.

8. Holder $\mathrm{N}$ and Klein R: Eph receptors and ephrins: effectors of morphogenesis. Development 126: 2033-2044, 1999.

9. Andres A-C and Ziemiecki A: Eph and ephrin signalling in mammary gland morphogenesis and cancer. J Mamm Gland Biol Neopl 8: 475-486, 2003.

10. Pasquale EB: EPH receptor signalling casts a wide net on cell behaviour. Nat Rev Mol Cell Biol 6: 462-475, 2005.

11. Dodelet VC and Pasquale EB: Eph receptors and ephrin ligands: embryogenesis to tumorigenesis. Oncogene 19: 1097-1101, 2000.

12. Hattori M, Osterfield M and Flanagan JG: Regulated cleavage of contact-mediated axon repellent. Science 289: 1360-1365, 2000 .

13. Zimmer M, Palmer A, Kohler J and Klein R: EphB-ephrin-B bi-directional endocytosis terminates adhesion allowing contact mediated repulsion. Nat Cell Biol 5: 869-878, 2003. 
14. Marston DJ, Dickinson S and Nobes CD: Rac dependent transendocytosis of ephrinBs regulates Eph-ephrin contact repulsion. Nat Cell Biol 5: 879-888, 2003.

15. Lackmann $M$ and Boyd AW: Eph, a protein family coming of age: more confusion, insight or complexity? Sci Signal 1: re2, 2008.

16. Murai KK and Pasquale EB: 'Eph' ective signalling: forward, reverse and crosstalk. J Cell Sci 116: 2823-2832, 2003.

17. Janes PW, Adikari S and Lackmann M: Eph/ephrin signalling and function in oncogenesis: lessons from embryonic development. Curr Cancer Drug Targets 8: 473-479, 2008.

18. Adams RH: Molecular control of arterial-venous blood vessel identity. J Anat 202: 105-112, 2003.

19. Andres A-C, Reid HH, Zürcher G, Blaschke R, Albrecht D and Ziemiecki A: Expression of two novel eph-related receptor tyrosine kinases in mammary gland development and carcinogenesis. Oncogene 9: 1461-1467, 1994.

20. Shin D, Garcia-Gardena G, Hayashi S-I, Gerety S, Asahara T, Stavrakis G, Isner J, Folkmann J, Gimbrone MA and Anderson DJ: Expression of ephrin-B2 identifies a stable genetic difference between arterial and venous vascular smooth muscle cells as well as endothelial cells and marks subsets of microvessels at sites of neovascuarisation. Dev Biol 230: 139-150, 2001 .

21. Batlle E, Bacani J, Begthel H, et al: EphB receptor activity suppresses colorectal cancer progression. Nature 435: 1126-1130, 2005 .

22. Zhao C, Irie N, Takada Y, Shimoda K, Miyamoto T, Nishiwaki T, Suda T and Matsuo K: Bidirectional ephrin-B2-EphB4 signalling controls bone homeostasis. Cell Metab 4: 111-121, 2006 .

23. Okubo T, Yanai $\mathrm{N}$ and Obinata $\mathrm{M}$ : Stromal cells modulate ephrin-B2 expression and transmigration of hematopoietic cells. Exp Hematol 34: 330-338, 2006.

24. Pasquale EB: Eph-ephrin bidirectional signalling in physiology and disease. Cell 133: 38-52, 2008.

25. Nikolova Z, Djonov V, Zürcher G, Andres A-C and Ziemiecki A: Cell-type specific and estrogen dependent expression of the receptor tyrosine kinase EphB4 and ist ligand ephrin-B2 during mammary gland morphogenesis. J Cell Sci 111: 2741-2751, 1998.

26. Munarini N, Jäger R, Abderhalden S, Zürcher G, Rohrbach V, Lörcher S, Pfanner-Meyer B, Andres A-C and Ziemiecki A: Altered mammary epithelial development, pattern formation and involution in transgenic mice expressing the EphB4 receptor tyrosine kinase. J Cell Sci 115: 25-37, 2002.

27. Andres A-C, Bchini O, Schubaur B, Dolder B, LeMeur M and Gerlinger P: Ha-ras induced transformation of mammary epithelium is favoured by increased oncogene expression or inhibition of mammary regression. Oncogene 6: 771-779, 1991.

28. Küng P, Nikolova Z, Djonov V, Hemphill A, Rohrbach V, Boehlen D, Zürcher G, Andres A-C and Ziemiecki A: A novel family of serine/threonine kinases participaring in spermiogenesis. J Cell Biol 139: 1851-1859, 1997.

29. Djonov V, Schmid M, Tschanz AA and Burri PH: Intussusceptive angiogenesis: its role in embryonic vascular network formation. Circ Res 86: 286-292, 2000.

30. Hennighausen L: Mouse models for breast cancer. Oncogene 19: 966-967, 2000

31. Cowan CA, Yokoyama N, Saxena A, Chumley MJ, Silvany RE, Baker LA, Srivastava D and Henkemeyer M: Ephrin-B2 reverse signalling is required for axon pathfinding but not early vascular development. Dev Biol 271: 263-271, 2004

32. Fuller T, Korff T, Kilian A, Dandekar G and Augustin HG: Forward EphB4 signalling in endothelial cells controls cellular repulsion and segregation from ephrin-B2 positive cells. J Cell Sci 116: 2461-2470, 2003

33. Adams RH, Diella F, Hennig S, Helmbacher F, Deutsch U and Klein R: The cytoplasmic domain of the ligand ephrin-B2 is required for vascular morphogenesis but not cranial neural crest migration. Cell 104: 57-69, 2001.

34. LaMarca $\mathrm{H}$ and Rosen J: Minireview: Hormones and mammary cell fate - what will I become when I grow up? Endocrinology 149: 4317-4321, 2008 .
35. Kullander K and Klein R: Mechanisms and functions of Eph and ephrin signalling. Nat Rev 3: 475-486, 2002.

36. Schatzmann F, Marlow R and Streuli C: Integrin signalling and mammary gland function. J Mamm Gland Biol Neopl 8: 395-408, 2003.

37. Jackson D, Bresnick J and Dickson C: A role for fibroblast growth factor signalling in the lobuloalveolar development of the mammary gland. J Mamm Gland Biol Neopl 2: 385-392, 1997.

38. Meniel V and Clarke AR: Wnt-cadherin connections in normal and neoplastic mammary epithelium. J Mamm Gland Biol Neopl 8: 435-448, 2003

39. Daniel CW, Strickland $P$ and Freemann $Y$ : Expression and functional role of $\mathrm{E}$ - and P-cadherins in mouse mammary ductal morphogenesis and growth. Dev Biol 169: 511-519, 1995.

40. Orsulic S and Kemler R: Expression of Eph receptors and ephrins is differentially regulated by E-cadherin. J Cell Sci 113: 1793-1802, 2000

41. Zantek ND, Walker-Daniels J, Stewart J, Hansen RK, Robinson D, Miao H, Wang B, Kung HJ, Bisselll MJ and Kinch MS: MCF-10A-neoST: a new cell system for studying cell-ECM and cell-cell interactions in breast cancer. Clin Cancer Res 7: 3640-3648, 2001 .

42. Boussaida O, Kutsch S, Hierholzer A, Delmas V and Kemler R: E-cadherin is a survival factor for the lactating mouse mammary gland. J Mech Dev 115: 53-62, 2002.

43. Héroult M, Schaffner F and Augustin HG: Eph receptor and ephrin ligand-mediated interactions during angiogenesis and tumor progression. Exp Cell Res 312: 642-650, 2006.

44. Zisch AH, Zeisberger SM, Ehrbar M, Djonov V, Weber CC, Ziemiecki A, Pasquale EB and Hubbell JA: Engineered fibrin matrices for functional display of cell membrane-bound growth factor-like activities: study of angiogenic signalling by ephrin-B2. Biomaterials 25: 3245-3257, 2004.

45. Malathy P, Shekar, V, Werdell J and Tait L: Interaction with endothelial cells is a prerequisite for branching-ductal-alveolar morphogenesis and hyperplasia of preneoplastic human breast epithelial cells: regulation by estrogen. Cancer Res 60: 439-449, 2000.

46. Shekar MP, Werdell J, Santner SJ, Pauley RJ and Tait L: Breast stroma plays a dominant regulatory role in breast epithelial growth and differentiation: implications for tumor development and progression. Cancer Res 61: 1320-1326, 2001

47. Noren NK, Lu M, Freeman AL, Koolpe M and Pasquale EB: Interplay between EphB4 on tumor cells and vascular ephrin-B2 regulates tumor grpwth. Proc Natl Acad Sci USA 110: 5583-5588, 2004.

48. Noren NK, Foos G, Hauser CA and Pasquale EB: The EphB4 receptor suppresses breast cancer cell tumorigenicity through an Abl-Crk pathway. Nat Cell Biol 8: 815-825, 2006

49. Alam SM, Fujimoto J, Jahan I, Sato E and Tamaya T: Coexpression of EphB4 and ephrin-B2 in tumor advancement of ovarian cancer. Br J Cancer 98: 845-851, 2008.

50. Alam SM, Fujimoto J, Jahan I, Sato E and Tamaya T: Overexpression of ephrin-B2 and EphB4 in tumor advancement of uterine endometrial cancer. Ann Oncol 18: 485-490, 2007

51. Berclaz G, Karamitopoulou E, Mazzucchelli L, Rohrbach V, Dreher E, Ziemiecki A and Andres A-C: Activation of the receptor tyrosine kinase EphB4 in endometrial hyperplasia and endometrial carcinoma. Ann Oncol 14: 220-226, 2003.

52. Walker-Daniels J, Hess AR, Hendrix MJ and Kinch MS: Differential regulation of EphA2 in normal and malignant cells. Am J Pathol 162: 1037-1042, 2003.

53. Zantek ND, Azimi M, Fedor-Chaiken M, Wang B, Brackenbury R and Kinch MS: E-cadherin regulates the function of the EphA2 receptor tyrosine kinase. Cell Growth Differ 10: 629-638, 1999.

54. Zelinsky DP, Zantek ND, Stewart JC, Irizarry AR and Kinch MS: EphA2 overexpression causes tumorigenesis of mammary epithelial cells. Cancer Res 61: 2301-2306, 2001.

55. Ogawa K, Pasqualini R, Lindberg RA, Kain R, Freeman AR and Pasquale EB: The ephrin-A1 ligand and its receptor, EphA2, are expressed during tumor neovascularization. Oncogene 19: 6043-6052, 2000 